\title{
The effects of vitamin $C$ supplementation on incident and progressive knee osteoarthritis: a longitudinal study
}

\author{
Jennifer Peregoy ${ }^{1}$ and Frances Vaughn Wilder ${ }^{2, *}$ \\ 'Department of Epidemiology, College of Public Health, University of South Florida, Clearwater, FL, USA: \\ ${ }^{2}$ Department of Epidemiology, College of Public Health, The Arthritis Research Institute of America, University of \\ South Florida, 300 S. Duncan Avenue Suite \#188, Clearwater, FL 33755, USA
}

Submitted 3 July 2009: Accepted 13 May 2010: First published online 16 August 2010

\begin{abstract}
Objective: To evaluate the association between vitamin $\mathrm{C}$ supplementation and the incidence and progression of radiographic knee osteoarthritis (OA).

Design: Prospective cohort study.

Setting: Clearwater Osteoarthritis Study (COS): (1988 to the present) a longitudinal study.

Subjects: Male and female COS participants aged 40 years and above ( $n$ 1023). The study exposure was the participants' self-reported history of vitamin $\mathrm{C}$ supplementation. The participants underwent biennial, sequential knee radiographs, which were assessed using the Kellgren-Lawrence ordinal scale to determine evidence of the study 2 outcomes: incident radiographic knee OA (RKOA) and progression of RKOA. Results: Individuals without baseline knee OA who self-reported vitamin C supplement usage were $11 \%$ less likely to develop knee OA than were those individuals who self-reported no vitamin $\mathrm{C}$ supplement usage (risk ratio $(\mathrm{RR})=0 \cdot 89,95 \% \mathrm{CI}$ 0.85, 0.93). Among those participants with RKOA at baseline, vitamin C supplement usage did not demonstrate an association with RKOA progression ( $\mathrm{RR}=0 \cdot 94,95 \%$ CI $0 \cdot 79,1 \cdot 22$ ).

Conclusions: In the present prospective cohort study, we found no evidence to support a protective role of vitamin $\mathrm{C}$ in the progression of knee OA. However, after controlling for confounding variables, these data suggest that vitamin $\mathrm{C}$ supplementation may indeed be beneficial in preventing incident knee OA. Given the massive public health burden of OA, the use of a simple, widely available and inexpensive supplement to potentially reduce the impact of this disease merits further consideration.
\end{abstract}

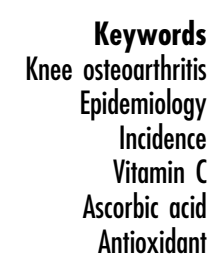

Damage caused by free radicals has long been thought to be pathogenic and they play an important role in the progression of many chronic diseases, including CVD and osteoarthritis $(\mathrm{OA})^{(1-6)}$. Free radicals are unstable, reactive molecules with unpaired electrons that are primarily created via the aerobic metabolism and the immune response to antigen. In the absence of sufficient antioxidants, free radicals can wreak havoc on neighbouring cells, causing cytotoxicity and cellular damage ${ }^{(7)}$. In fact, although OA is defined as a 'non-inflammatory arthropathy', there is evidence that pro-inflammatory molecules play an important role as mediators in the pathogenesis of $\mathrm{OA}^{(8,9)}$. Thus, there is significant scientific interest in studying the efficacy of nutritional therapeutics to combat such chronic diseases, specifically dietary antioxidants such as vitamin C. In addition to its antioxidant capacity, vitamin $\mathrm{C}$ is critical to bone health, acting as an electron donor in the synthesis of type II collagen ${ }^{(4-6)}$. It is also a sulfate carrier in glycosaminoglycan synthesis. This may be relevant to OA aetiology because the depletion of sulfated proteoglycans from the articular cartilage extracellular matrix is one of the earliest expressions of $\mathrm{OA}$, eventually resulting in cartilage degeneration $^{(6)}$. Vitamin $\mathrm{C}$ deficiency may therefore be a risk factor in the development of OA, leading to the logical possibility of using vitamin $\mathrm{C}$ supplementation for primary prevention or as a therapeutic intervention for OA. The present study followed a group of participants free of radiographic knee OA (RKOA) to quantify the role of vitamin $\mathrm{C}$ supplement usage in incident RKOA. In addition, among a group of participants with RKOA at baseline, we examined the relationship between vitamin $\mathrm{C}$ supplement usage and RKOA progression.

\section{Methods}

The study hypotheses were: (i) among those without RKOA at study baseline, the likelihood of developing 
RKOA is lower among those participants reporting vitamin $\mathrm{C}$ supplement usage than among those who report no vitamin C supplement usage; and (ii) among those with RKOA at baseline, the likelihood of RKOA progression is lower among those participants reporting vitamin $\mathrm{C}$ supplement usage than among those who report no vitamin C supplement usage. The two study outcomes were RKOA incidence and RKOA progression. The study exposure, vitamin $\mathrm{C}$ supplementation, was defined as the cumulative number of years of self-reported vitamin $\mathrm{C}$ supplement usage since study enrolment.

\section{Subjects}

The Clearwater Osteoarthritis Study (COS), initiated by The Arthritis Research Institute of America in 1988, is an on-going community-based, prospective cohort study with over 3700 participants (aged $>40$ years). The research objective of the COS is to identify the major risk factors for the development and progression of OA and to differentiate risk factors for localised and generalised primary OA. Data are collected from study participants biennially, and include demographic, historical, clinical and radiological information. COS volunteer recruitment is conducted using a variety of methods, including invitational letters, television and radio announcements, newspaper articles publicising the COS study, articles posted in community organisations' bulletins, as well as seminars held at community clubs and organisations. To ensure a representative sample that includes younger participants who are more likely to be free of OA, recruitment efforts were used to encourage participation by employees of the Pinellas County School System, the City of Clearwater and Pinellas County Inc.

\section{Data collection}

During the initial visit, a detailed study description was given to the participant, followed by a screening questionnaire to determine eligibility based on predetermined inclusion/exclusion criteria. Exclusion criteria were limited to individuals with self-reported rheumatoid arthritis or variants (lupus erythematosus, ankylosing spondylitis, etc.), gout, disabling neuralgic disease, confined to a wheelchair or mentally incompetent. Once eligibility was established, informed consent was obtained before data collection. All eligible, voluntary participants completed the 'COS history questionnaire' and underwent a physical examination. This examination included radiographs of four sites including the anterior-posterior weightbearing knee, taken by a licensed X-ray technician using standardised exposure techniques. Study participants were re-evaluated biennially, updating the history questionnaire and the clinical examination information.

\section{Radiographic measurement}

Radiographs were interpreted by a board-certified radiologist, blinded to the individual study participants' status. Using the Kellgren-Lawrence ordinal scale ${ }^{(10)}$, knee OA cases were defined as those with grades $>2$, whereas those with grades 0 or 1 were considered disease-free. An 'incident' case was defined as a participant who was free of RKOA in both knees at study baseline, but developed RKOA in either one or both knees during the observation period. A progression case was defined as a study participant (with uni- or bilateral RKOA at baseline) who experienced the outcome of RKOA progression (of $\geq 1$ grade) during the follow-up period. Quality control measures were taken to ensure the validity of the radiographic assessments. Every tenth participant's assembled radiographs were independently interpreted by a non-affiliated radiologist (blinded to initial reading results), allowing for the quantification of interobserver variability. This was calculated using the $\kappa$ coefficient, measuring the extent of reader agreement above than due to chance alone ${ }^{(11)}$. Inter-reader reliability by a second radiologist reflected $93 \%$ agreement $(\kappa=0 \cdot 85)$.

\section{Questionnaire}

Information regarding the history of the participants' vitamin C supplement usage was collected using the 'COS history questionnaire'. Participants who indicated vitamin $\mathrm{C}$ supplement usage were further queried with 'Age started?' and 'Age stopped?' The COS database was being explored for its nutrition-related variables. A recently published COS study suggested a potentially protective relationship between multivitamin usage and the risk of the development and progression of $\mathrm{OA}^{(12)}$. To best assess solely the role of vitamin $\mathrm{C}$, all participants who reported a history of multivitamin usage were excluded from the present analyses.

In an effort to identify and control for potential confounders, several variables associated with RKOA and vitamin C supplementation were investigated. Potential confounders investigated were age, gender, baseline BMI, history of knee injury, exercise status and total years of vitamin C supplement usage before study baseline. Participants' history of knee injury was a self-reported yes/no variable. The questionnaire asked 'Have you ever been told by a doctor that you have a cracked, fractured, broken or dislocated bone?' If the participant answered yes, they were asked to select from a list of body sites (e.g. knee) to indicate where they sustained an injury. Exercisers were defined as those participants who self-reported an exercise frequency of at least three times weekly (for minimum of $20 \mathrm{~min}$ per session). For the present analyses, confounders were defined as any factors associated with the outcome (RKOA incidence/RKOA progression) and differentially distributed by exposure status (vitamin $\mathrm{C}$ supplementation since study enrolment). Baseline BMI, age and prior years of vitamin $C$ supplement usage were included in the adjusted analysis as continuous variables.

\section{Statistical analyses}

With 866 participants classified as eligible for developing RKOA, the present study had an $80 \%$ power to detect a 
$20 \%$ or greater difference in RKOA incidence by vitamin C supplement usage. Although the study of RKOA progression was limited to 157 participants, this sample size was also sufficient to allow for $80 \%$ power to detect a

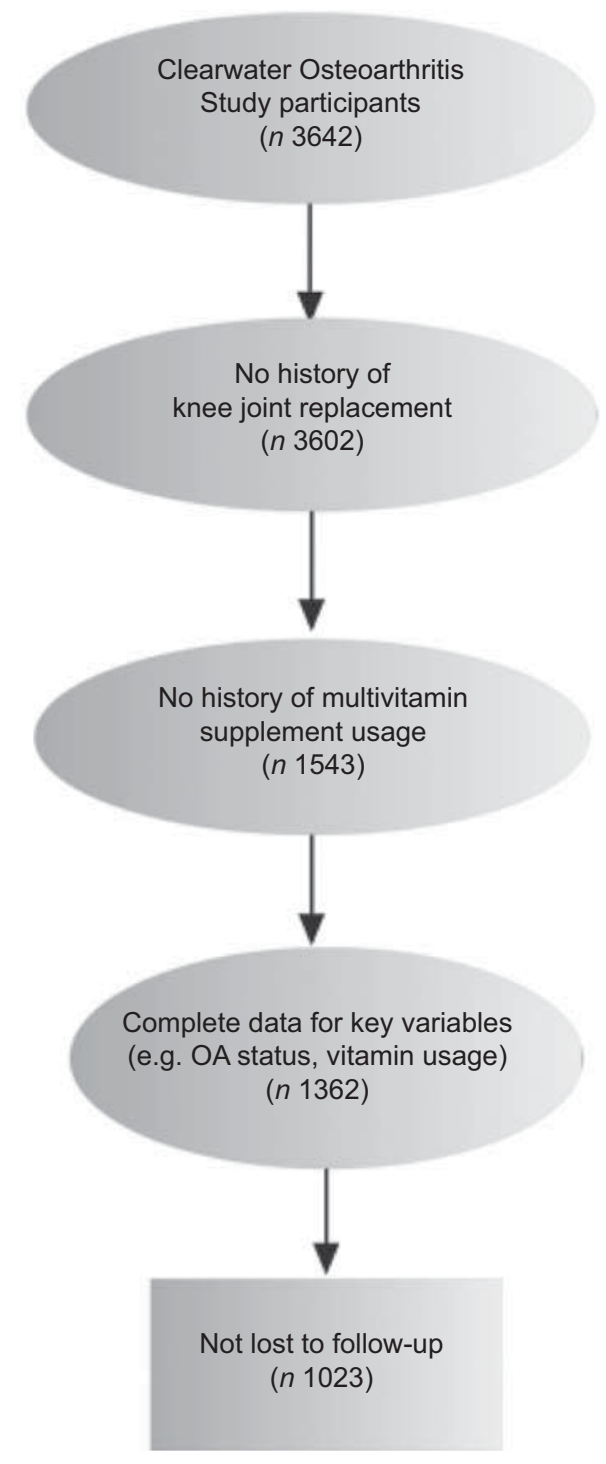

Fig. 1 Sample selection from the Clearwater Osteoarthritis Study (n 1023). OA, osteoarthritis
$20 \%$ difference in RKOA progression by vitamin C status, if indeed a difference existed (two-tailed; $\alpha=0 \cdot 05)^{(13)}$.

Given the unequal follow-up times and censored participants inherent in a cohort study design, the Cox proportional hazards model was applied ${ }^{(14,15)}$ to quantify the relationship between vitamin $\mathrm{C}$ supplement usage and RKOA while simultaneously controlling for the influence of other factors discussed above. The Cox regression model was also used to calculate the unadjusted risk ratios (RR), with vitamin $\mathrm{C}$ supplementation as the sole independent variable. The study observation period was the interval between study enrolment and either (i) the development of RKOA; (ii) study withdrawal; or (iii) censoring. Results were expressed as RR (hazard ratios), along with the corresponding 95\% CI and $P$ values. $P<0.05$ was considered statistically significant. The data analyses were conducted using the SAS statistical software package version $9 \cdot 13$ (SAS Institute, Cary, NC, USA), specifically applying PROC PHREG ${ }^{(16)}$.

\section{Results}

The final sample size was 1023. Figure 1 displays the participant selection process. Of the 866 participants free of RKOA at baseline, 341 (39\%) developed RKOA during the observation period. Among the 157 study participants with RKOA at baseline, fifty-one (32\%) experienced RKOA progression during the observation period. The mean period of observation among those with and without baseline RKOA was $7 \cdot 5$ (SD 5.3) and $6 \cdot 1$ (SD 4.4) years, respectively. The average time between physical examinations for RKOA progression and incident cohorts was $2 \cdot 08$ (SD $0 \cdot 39)$ and $2 \cdot 12(\mathrm{sD} 0 \cdot 42)$ years, respectively. Table 1 summarises study sample characteristics for the RKOA incidence and progression analyses.

The unadjusted RR, generated using Cox's regression with vitamin C supplement usage as the sole independent variable, quantified the association between vitamin $\mathrm{C}$ supplement usage and incident RKOA. These data indicated that those individuals reporting vitamin $\mathrm{C}$ supplement usage were $7 \%$ less likely to develop RKOA than those individuals who reported no vitamin $\mathrm{C}$ supplement

Table 1 Study sample baseline characteristics (by radiographic knee osteoarthritis status)

\begin{tabular}{|c|c|c|c|c|}
\hline & \multicolumn{2}{|c|}{$\begin{array}{l}\text { Incidence analyses } \\
(n \text { 866) }\end{array}$} & \multicolumn{2}{|c|}{$\begin{array}{l}\text { Progression analyses } \\
(n 157)\end{array}$} \\
\hline & $n$ & $\%$ & $n$ & $\%$ \\
\hline Female & 553 & $63 \cdot 9$ & 88 & $56 \cdot 1$ \\
\hline Knee injury history (yes) & 390 & $45 \cdot 0$ & 76 & $48 \cdot 4$ \\
\hline \multirow[t]{2}{*}{ Exercise status (yes) } & 291 & $37 \cdot 9$ & 49 & $36 \cdot 8$ \\
\hline & Average & SD & Average & SD \\
\hline BMI $\left(\mathrm{kg} / \mathrm{m}^{2}\right)$ & $25 \cdot 6$ & $4 \cdot 0$ & $28 \cdot 1$ & $5 \cdot 6$ \\
\hline Age (years) & $61 \cdot 1$ & $10 \cdot 6$ & $66 \cdot 5$ & $8 \cdot 7$ \\
\hline Total years of vitamin $C$ supplement usage before study baseline & $3 \cdot 2$ & $9 \cdot 1$ & $2 \cdot 8$ & $9 \cdot 7$ \\
\hline
\end{tabular}


Table 2 Radiographic knee osteoarthritis and vitamin C supplement usage

\begin{tabular}{lcclll}
\hline & \multicolumn{2}{c}{ Unadjusted } & & \multicolumn{2}{c}{ Adjustedt } \\
\cline { 2 - 3 } \cline { 6 - 7 } & $\mathrm{RR}$ & $95 \% \mathrm{Cl}$ & & $\mathrm{RR}$ & $95 \% \mathrm{Cl}$ \\
\hline Incidence & 0.93 & $0.89,0.96^{*}$ & & 0.89 & $0.85,0.93^{*}$ \\
Progression & 0.91 & $0.79,1.04$ & & 0.94 & $0.79,1.12$ \\
\hline
\end{tabular}

${ }^{*} P$ value $<0.0001$

† Adjusted for BMI, gender, age, knee injury history, exercise and vitamin C supplement usage before study baseline.

usage ( $\mathrm{RR}=0 \cdot 93,95 \% \mathrm{CI} 0 \cdot 89$, 0.96; Table 2). Adjusted analyses simultaneously considered the possible effects of baseline BMI, gender, age, knee injury history, exercise status and total years of vitamin $\mathrm{C}$ supplement usage before study baseline. The adjusted association between vitamin C supplement usage and incident RKOA demonstrated that those individuals reporting vitamin $\mathrm{C}$ supplement usage were $11 \%$ less likely to develop RKOA than those individuals who reported no vitamin $\mathrm{C}$ supplement usage $(\mathrm{RR}=0 \cdot 89,95 \% \mathrm{CI} 85,0 \cdot 93$; Table 2$)$.

Among those participants with baseline RKOA, the unadjusted RR quantified the association between vitamin $\mathrm{C}$ supplement usage and the subsequent progression of RKOA. These data did not demonstrate a statistically significant relationship between vitamin $\mathrm{C}$ supplement usage and RKOA progression ( $\mathrm{RR}=0.91,95 \%$ CI $0 \cdot 79$, $1 \cdot 04, P$ value $=0 \cdot 15$; Table 2 ). The adjusted risk estimate assessing vitamin $\mathrm{C}$ supplement usage and RKOA progression did not indicate a relationship between vitamin $\mathrm{C}$ supplement usage and RKOA progression $(\mathrm{RR}=0.94$, $95 \%$ CI $0 \cdot 79,1 \cdot 12, P$ value $=0 \cdot 48$; Table 2 ) .

\section{Discussion}

This epidemiological investigation demonstrated an association between vitamin $\mathrm{C}$ supplement usage and a reduced incidence of RKOA. These findings are supported by a cross-sectional study conducted in 2007 , examining the effect of antioxidant intake on knee cartilage and bone in healthy individuals $(n \text { 293 })^{(17)}$. Antioxidant intake was estimated from a baseline FFQ. MRI of the knee was taken at baseline and again at 10 years. Wang et $a l .{ }^{(17)}$ found vitamin $\mathrm{C}$ intake to be associated with a $50 \%$ risk reduction of bone marrow lesions (OR = $0 \cdot 50,95 \%$ CI $0 \cdot 29,0 \cdot 87, P=0 \cdot 01)$ and inversely associated with tibial plateau bone area $(\beta=-35 \cdot 5 \%$, 95\% $\mathrm{CI}=-68 \cdot 8,-2 \cdot 3, P=0 \cdot 04)$, both of which are involved in the pathogenesis of knee OA. Increased vitamin $C$ intake has also been shown to exhibit a slight chondroprotective effect $(P=0 \cdot 08)$ on the development of spontaneous lesions in guinea pigs, with no such effect on the progression of surgically induced $\mathrm{OA}^{(18)}$. The findings of Kurz et $a l^{(4)}$ demonstrated a similar positive association between antioxidant supplements (vitamins A,
$\mathrm{C}, \mathrm{E}, \mathrm{B}_{6}, \mathrm{~B}_{2}$ and selenium) and reduced incidence of knee $\mathrm{OA}$ in OA-prone mice. It was found that the supplemented diet induced a $1 \cdot 5$-fold increase in enzymatic antioxidant activity, with the authors concluding that the selected combination of vitamins and selenium diminished the mechanical induction of OA in mice. However, given the multivitamin supplementation strategy used in that study, it is difficult to conclude which of these nutrients may have had the largest influence or whether synergistic effects played a role.

However, among those with RKOA, the present study found no statistically significant association between vitamin C supplement usage and reduced progression of RKOA. Some previously published, related clinical studies have reported opposite findings. In the Framingham Osteoarthritis Study, a threefold risk reduction in OA progression was observed in both the middle tertile $(\mathrm{OR}=0 \cdot 3,95 \% \mathrm{CI} 0 \cdot 1,0 \cdot 8)$ and highest tertile $(\mathrm{OR}=0 \cdot 3$, $95 \%$ CI $0 \cdot 1,0 \cdot 6$ ) of vitamin $\mathrm{C}$ intake, yet no association was found between vitamin $\mathrm{C}$ and incident $\mathrm{OA}^{(5)}$. McAlindon et $a l .{ }^{(5)}$ also noted that those with the highest vitamin $\mathrm{C}$ intake experienced a reduced risk of developing knee pain $(\mathrm{OR}=0 \cdot 3,95 \% \mathrm{CI} 0 \cdot 1,0 \cdot 8)$. Reduction in OA knee pain associated with vitamin $\mathrm{C}$ supplementation has been established in several clinical investigations, such as those recently conducted by Jensen et $a l^{(19)}$ and Baker et al. ${ }^{(20)}$. Although the Framingham Osteoarthritis Study was rigorous in its design and execution, the study used a relatively small sample size ( $n 81$ for incident OA; $n 68$ for progressive OA). Similar results demonstrated in the MOST study found that those with the lowest serum vitamin $\mathrm{C}$ concentration were at a twofold greater risk of further OA progression as compared to those in the highest quartile $(\mathrm{OR}=2 \cdot 12,95 \% \mathrm{CI} 1 \cdot 30,3 \cdot 46, P=0 \cdot 003)$, yet found no statistically significant association between serum vitamin $\mathrm{C}$ and knee OA incidence ( $n 156$ for incident OA; $n 408$ for progressive OA) ${ }^{(21)}$.

Vitamin C status has been shown to influence the progression of OA in animal models as well. In a study conducted by Schwartz et $a l^{(22)}$, guinea pigs were supplemented with either low or high doses $(150 \mathrm{mg} / \mathrm{d})$ of ascorbate before surgically inducing knee OA. The animals on low dose were marked by more severe pathological changes than those seen in high-dose animals. The high-dose animals demonstrated higher cartilage retention in their normal joints, suggesting that the vitamin C stimulated additional collagen production. Schwartz et al. ${ }^{(22)}$ concluded that high doses of ascorbate have a protective effect on the severity of surgically induced knee OA in guinea pigs. Our findings failed to support such an association among participants with RKOA and self-reported vitamin C status. Hill and Bird ${ }^{(23)}$ also failed to report an association between seleniumACE supplementation and knee OA outcomes (pain, stiffness and radiographic evidence of disease progression). Although this was a rigorous 6-month, double-blind 
placebo-controlled trial, study limitations (including trace amounts of selenium found in the placebo), as well as low power due to small sample size ( $n$ 30) should be considered.

In contradiction to all other published findings, a 2004 study conducted on guinea pigs observed a dose-dependent, positive association between ascorbic acid supplementation and the severity of spontaneous $\mathrm{OA}^{(24)}$. In addition, Kraus et al. ${ }^{(24)}$ noted a correlation between the knee joint histological severity scores and plasma ascorbate concentration ( $r=0 \cdot 38, P=0 \cdot 01)$ and concluded that osteoarthritic patients should not consume above the Recommended Daily Value for vitamin C. The present study highlights the complexity of the relationship between the pathogenesis of OA and dietary antioxidant consumption and underscores the need for related, rigorous clinical research investigations.

The association demonstrated between vitamin $\mathrm{C}$ supplementation and the reduced risk of incident RKOA in our study, while inconsistent with data from some published studies, is biologically plausible given the current understanding of the physiological effects of vitamin $\mathrm{C}$ intake on bone health and the natural history of OA. It has been commonly hypothesised that mechanical stress on the joints may lead to free radical accumulation and subsequent articular cartilage degradation, leading to OA symptoms ${ }^{(4,25)}$. This presents the possibility that the consumption of antioxidants may reduce the incidence of OA by preventing or minimising the occurrence of oxidative cartilage damage. Evidence from both in vitro and animal model studies supports this hypothesis.

The chondrocyte in normal human cartilage maintains a metabolic homeostasis, balancing between anabolism and catabolism of the extracellular matrix. However, in chondrocytes of OA-affected individuals, this balance is skewed in favour of catabolism, resulting in matrix degradation and eventual loss of the articular cartilage that leads to OA symptoms and progression ${ }^{(26,27)}$. Thus, a major focus of $\mathrm{OA}$ research has been on the mechanism of the chondrocyte in the OA disease pathway. The findings of Yudoh et $a l^{(28)}$ demonstrated that oxidative stress induces genomic instability and dysfunction of human chondrocytes in OA cartilage, with ascorbic acid mitigating this effect. This suggests that oxidative stress may indeed play a significant role in the cartilage degradation associated with the development of OA, with implications that antioxidants such as vitamin $\mathrm{C}$ may reduce this risk. Similarly, vitamin C supplementation has been demonstrated to reduce in vitro chondrocyte degeneration, marked by reduced morphological degradation and higher collagen content, after static loading ${ }^{(29)}$.

Cartilage metabolism, a step in the OA disease pathway, has been demonstrated to be directly affected by vitamin C status in animal models. Peterkovsky et al. ${ }^{(30)}$ observed decreased cartilage collagen synthesis in vitamin C-deficient guinea pigs, while conversely, the addition of ascorbate to guinea pig articular cartilage explants has been shown to increase the synthesis of collagen and aggrecan $^{(31)}$. Although classic signs of inflammation are absent in OA (e.g. neutrophils in synovial fluid), there is significant evidence that suggests that inflammatory molecules may be important mediators in the OA disease pathway ${ }^{(27,32)}$. For instance, inflammatory cytokines such as IL-1 have been found in the synovial fluid of OA patients and are known to stimulate catabolic processes such as the synthesis of proteolytic enzymes. Research has shown that chondrocytes of OA-afflicted individuals exhibit enhanced expression of pro-inflammatory cytokines and inducible NO synthase, the molecule that stimulates the production of NO, a strong oxidising agent ${ }^{(27)}$. These inflammatory cytokines have been linked to the inhibition of cartilage proteoglycan and collagen synthesis, as well as chondrocyte apoptosis ${ }^{(27,32)}$. If indeed these autocrine/paracrine inflammatory pathways play a pathogenic role in the development or progression of $\mathrm{OA}$, it is reasonable to believe that antioxidant supplements such as vitamin C may prove to be a powerful tool in the primary or secondary prevention of this disease. Vitamin C as an anti-inflammatory molecule is virtually unrivalled in its potency. Although studies conducted on the role of vitamin C in OA pathology have yielded conflicting results, it continues to be an active area of research that merits further investigation.

\section{Study strengths and limitations}

The present study's prospective cohort design is able to clearly establish the temporal relationship between vitamin C supplement usage and the subsequent development of RKOA. A strength of the present study is the collection of serial radiographs for all participants beginning at study entry, allowing us to determine pre-existing RKOA disease status among the study participants. Loss to follow-up is always of concern in any prospective cohort design. Differences by selected characteristics between those participants who were lost to follow-up and those who were not lost were examined (Table 3). Overall, it can be seen that the two groups, lost and retained, had similar baseline characteristics. There were no significant differences between the two groups by age, sex, BMI, knee injury status or exercise status. However, the total years of vitamin C supplement usage before study baseline among the participants lost to follow-up was half that of the retained participants. Although our adjusted analyses included this factor, it is possible that the study results may have been biased away from the null.

A study limitation is the self-reported nature of the exposure, vitamin $\mathrm{C}$ supplement usage. Whenever selfreported data are collected, there exists the possibility of misclassification. Yet the prospective cohort design with biennial examinations minimised the risk of misclassification due to recall bias. In addition, ascribing an association of vitamins and better health to a causal path may be fraught with problems. Persons who regularly take vitamin 
Table 3 Evaluation of losses to follow-up by baseline characteristics (vitamin C supplement usage and radiographic knee OA)

\begin{tabular}{|c|c|c|c|c|}
\hline & \multicolumn{2}{|c|}{ Incidence analyses } & \multicolumn{2}{|c|}{ Progression analyses } \\
\hline & $\begin{array}{l}\text { Retained } \\
\text { (n 866) }\end{array}$ & $\begin{array}{c}\text { Lost } \\
\text { (n 272) }\end{array}$ & $\begin{array}{l}\text { Retained } \\
(n \text { 157) }\end{array}$ & $\begin{array}{l}\text { Lost } \\
(n 67)\end{array}$ \\
\hline Female & $63 \cdot 9$ & $63 \cdot 2$ & $56 \cdot 1$ & $59 \cdot 7$ \\
\hline Knee injury history (yes) & $45 \cdot 0$ & $50 \cdot 0$ & $48 \cdot 4$ & $46 \cdot 3$ \\
\hline Exercise status (yes) & $37 \cdot 9$ & $35 \cdot 7$ & $36 \cdot 8$ & $37 \cdot 9$ \\
\hline Baseline knee OA score $=2$ & N/A & N/A & $65 \cdot 0$ & $50 \cdot 8$ \\
\hline Baseline knee OA score $=3$ & $\mathrm{~N} / \mathrm{A}$ & $\mathrm{N} / \mathrm{A}$ & $21 \cdot 0$ & $23 \cdot 9$ \\
\hline BMI $\left(\mathrm{kg} / \mathrm{m}^{2}\right)$ & $25 \cdot 6$ & $25 \cdot 7$ & $28 \cdot 1$ & $29 \cdot 6$ \\
\hline Age (years) & $61 \cdot 1$ & $62 \cdot 5$ & $66 \cdot 5$ & $66 \cdot 5$ \\
\hline Total years of vitamin $\mathrm{C}$ supplement usage before study baseline & $3 \cdot 2$ & $1 \cdot 6$ & $2 \cdot 8$ & $1 \cdot 4$ \\
\hline
\end{tabular}

OA, osteoarthritis; N/A, not applicable.

C may routinely demonstrate health choices that impact their predisposition to OA. They may be more concerned with their health compared with persons who do not take vitamin C. Therefore, although a causal interpretation cannot be ruled out, it is by no means proven. As with any epidemiological study, confounding poses a threat to validity. Yet, appropriate measures were taken by applying restrictive inclusion/exclusion criteria and conducting multivariate analyses to address potential bias. The statistical power afforded by the large sample size is a strength of the present study. Although employing smaller sample sizes, related previously conducted studies provided the justification for our research. Our study builds on those findings. In addition, the inter-reader variability of the radiographic assessments was quite low, as demonstrated by the high $\kappa$ value. There is some controversy about OA diagnoses (e.g. symptomatic, radiographic, weight-bearing status, etc.). To assess OA incidence and progression, the present study employed the Kellgren-Lawrence scale, a fairly common approach with films read blindly using a standardised technique. Given that the KellgrenLawrence composite score relies heavily on osteophytes, it may be useful to examine the development of osteophytes and joint space narrowing separately to further elucidate the mechanism of action of vitamin $C$. The feasibility of the necessary research protocol modifications is being investigated to include such an assessment for future COS radiographic examinations.

In conclusion, new efforts to prevent the incidence and progression of radiographic OA may include strategies aimed at reducing oxidative damage in cartilage, thought to promote this disease. OA, the most common joint disease and a leading cause of disability, affects more than 27 million persons in the USA ${ }^{(33)}$. Vitamin C supplementation may very well be an effective strategy to combat this growing public health problem. However, there is at present a lack of substantial evidence to support this theory. There are strong inconsistencies found in both the study designs and outcomes, rendering it difficult to compare results across studies. The acceptable and most effective dosing range of vitamin $\mathrm{C}$ is still controversial, but recent studies have found that doses of up to $2000 \mathrm{mg} / \mathrm{d}$ are safe for most adults ${ }^{(33,34)}$. Clinicians may want to consider recommending vitamin $\mathrm{C}$ supplements to those at greatest risk for OA, as it has been shown to be safe within the normal dosing range and may offer additional health benefits beyond the hypothesised protection against OA. Multiple laboratory and clinical studies have shown promising results for the protective effect of vitamin $\mathrm{C}$ intake on the course of OA, including disease incidence, progression and symptomatology. Yet the heterogeneity of the related study findings provides evidence that further clinical studies are required to carefully investigate the safety and efficacy of the use of vitamin $\mathrm{C}$ in preventing or slowing the progression of OA.

\section{Acknowledgements}

The institute's research is supported by private funding. The authors have no conflicts of interest to declare. J.P. contributed to the literature review, data interpretation, manuscript writing and manuscript preparation; F.V.W. contributed to the study design, computer programming, data interpretation and manuscript writing.

\section{References}

1. Felson D \& Zhang Y (1998) An update on the epidemiology of knee and hip osteoarthritis with a view to prevention. Arthritis Rheum 41, 1343-1355.

2. Ameye L \& Chee W (2006) Osteoarthritis and nutrition. From nutraceuticals to functional foods: a systematic review of the scientific evidence. Arthritis Res Ther 8, R127.

3. Canter PH, Wider B \& Ernst E (2007) The antioxidant vitamins A, C, E and selenium in the treatment of arthritis: a systematic review of randomized clinical trials. Rheumatology 46, 1223-1233.

4. Kurz B, Jost B \& Schünke M (2002) Dietary vitamins and selenium diminish the development of mechanically induced osteoarthritis and increase the expression of antioxidative enzymes in the knee joint of STR/1N mice. Osteoarthritis Cartilage 10, 119-126.

5. McAlindon TE, Jacques P, Zhang YQ et al. (1996) Do antioxidant micronutrients protect against the development and progression of knee osteoarthritis? Arthritis Rheum 39, 648-656. 
6. Sowers M \& Lachance L (1999) Vitamins and arthritis - the roles of vitamins A, C, D, and E. Rheum Dis Clin North Am 25, 315-332.

7. Frei B (1994) Reactive oxygen species and antioxidant vitamins: mechanisms of action. Am J Med 97, 5S-13S; discussion 22S-28S

8. Pinto S, Rao AV \& Rao A (2008) Lipid peroxidation, erythrocyte antioxidants and plasma antioxidants in osteoarthritis before and after homeopathic treatment. Homeopathy 97, 185-189.

9. McAlindon TE (2006) Nutraceuticals: do they work and when should we use them? Best Pract Res Clin Rheumatol 20, 99-115.

10. Kellgren JH \& Lawrence JS (1963) Atlas of Standard Radiographs: The Epidemiology of Chronic Rheumatism, vol. 2. Oxford: Blackwell Scientific.

11. Kelsey JL, Thompson WD \& Evans AS (1986) Methods in Observational Epidemiology, pp. 290-292. New York: Oxford University Press.

12. Wilder FV, Leaverton PE, Rogers MW et al. (2009) Vitamin supplements and radiographic knee osteoarthritis: the Clearwater Osteoarthritis Study. J Musculoskel Res 12, 85-93.

13. Hulley SB \& Cummings SR (1988) Designing Clinical Research, pp. 139-150. Baltimore, MD: Williams \& Wilkins.

14. Bull K \& Speigelhelter DJ (1997) Tutorial in biostatistics: survival analysis in observational studies. Stat Med 16, 1040-1047.

15. Dawson-Saunders B \& Trapp RG (1994) Basic and Clinical Biostatistics, 2nd ed., pp. 221-222. Norwalk, CT: Appleton \& Lange.

16. SAS Institute Inc. (1990) SAS Procedures Guide, Ver 6, 3rd ed. Cary, NC: SAS Institute Inc.

17. Wang Y, Hodge AM, Wluka AE et al. (2007) Effect of antioxidants on knee cartilage and bone in healthy, middle-aged participants: a cross-sectional study. Arthritis Res Ther 9, R66.

18. Meacock SCR, Bodmer JL \& Billingham MEJ (1990) Experimental osteoarthritis in guinea pigs. J Exp Pathol 71, 279-293.

19. Jensen N (2003) Reduced pain from osteoarthritis in hip joint or knee joint during treatment with calcium ascorbate. A randomized, placebo-controlled cross-over trial in general practice. Ugeskr Laeger 165, 2563-2566.

20. Baker K, Niu JB, Goggins J et al. (2003) The effects of vitamin $\mathrm{C}$ intake on pain in knee osteoarthritis (OA). Arthritis Rheum 48, S422.
21. Chaganti RK, Tolstykh I, Javaid MK et al. (2008) Association of baseline vitamin $\mathrm{C}$ with incident and progressive radiographic knee OA. The MOST study. Arthritis Rheum 58, S897.

22. Schwartz ER, Leveille C \& Oh WH (1981) Experimentallyinduced osteoarthritis in guinea-pigs - effect of surgicalprocedure and dietary-intake of vitamin-C. Lab Anim Sci 31, 683-687.

23. Hill J \& Bird HA (1990) Failure of selenium-ACE to improve osteoarthritis. Br J Rbeumatol 29, 211-213.

24. Kraus VB, Huebner JL, Stabler T et al. (2004) Ascorbic acid increases the severity of spontaneous knee osteoarthritis in a guinea pig model. Arthritis Rheum 50, 1822-1831.

25. McAlindon T \& Felson DT (1997) Nutrition: risk factors for osteoarthritis. Ann Rheum Dis 56, 397-400.

26. Cotran RS, Kumar V \& Collins T (editors) (1999) Pathologic Basis of Disease, 6th ed. Philadelphia, PA: Saunders.

27. Hedbom E \& Hauselmann HJ (2002) Molecular aspects of pathogenesis in osteoarthritis: the role of inflammation. Cell Mol Life Sci 59, 45-53.

28. Yudoh K, van Trieu N, Nakamura H et al. (2005) Potential involvement of oxidative stress in cartilage senescence and development of osteoarthritis: oxidative stress induces chondrocyte telomere instability and downregulation of chondrocyte function. Arthritis Res Ther 7, R380-R391.

29. Sharma G, Saxena RK \& Mishra P (2008) Regeneration of static-load-degenerated articular cartilage extracellular matrix by vitamin C supplementation. Cell Tissue Res 334, 111-120.

30. Peterkofsky B (1991) Ascorbate requirement for hydroxylation and secretion of procollagen: relationship to inhibition of collagen synthesis in scurvy. Am J Clin Nutr 54, Suppl. 6, 1135S-1140S.

31. Clark AG, Rohrbaugh AL, Otterness I et al. (2002) The effects of ascorbic acid on cartilage metabolism in guinea pig articular cartilage explants. Matrix Biol 21, 175-184.

32. Goldring MB \& Goldring SR (2007) Osteoarthritis. J Cell Physiol 213, 626-634.

33. Lawrence RC, Felson DT, Helmick CG et al. for the National Arthritis Data Workgroup (2008) Estimates of the prevalence of arthritis and other rheumatic conditions in the United States: Part II. Arthritis Rheum 58, 26-35.

34. Hathcock J, Azzi A, Blumberg J et al. (2005) Vitamins E and $\mathrm{C}$ are safe across a broad range of intakes. Am J Clin Nutr 81, 736-745. 